Página inicial: 265 - Página Final: 280

Tipo de artículo: Reflexión derivada de investigación.

\title{
Evaluación Dialogante: fundamentos para un enfoque innovador en Educación Superior.
}

\author{
Dialogical Assessment: Foundations for an Innovative Approach in Higher \\ Education.
}

\author{
Por: Aida Josefina Rojas-Fajardo ${ }^{1}$
}

Recibido: junio de 2016 Revisado: noviembre de 2016 Aceptado: diciembre de 2016

\section{Resumen.}

Presentar una propuesta epistemológica que, de respuesta a los sentidos referidos por los mismos profesores frente a la evaluación profesoral, nos refiere, según resultados de este estudio, al diálogo como opción en la evaluación del desempeño profesoral, porque facilita el encuentro profesor-estudiante, es un espacio para la palabra, la reflexión, la democracia, lo ético, la construcción pedagógica y la construcción de sentidos y significados. Esta propuesta de evaluación dialogante, se basa en fundamentos empíricos, teóricos, pedagógicos, epistemológicos, ontológicos y éticos de la evaluación dialogante.

Palabras clave. Evaluación del docente, formación académica, comunicación interactiva, sentidos, construcción de sentidos.

\begin{abstract}
.
Putting forward an epistemological proposal that makes sense of the senses referred by the teachers themselves as for teachers' assessment, leads us, according to the results of the present study, to the dialog as an option for teachers' performance assessment because this makes the meeting between the teacher and the student easy. It is a space for the word, reflection, democracy, ethics, the pedagogic construction, and the construction of senses and meanings. This proposal of dialogical assessment is based on empirical, theoretical, pedagogic, epistemological, ontological, and ethical foundations of dialogical assessment.
\end{abstract}

Key words. Teachers' Assessment, Academic Training, Interactive Communication, Senses, and Construction of Senses.

1 Ph. D. en Ciencias de la Educación, Magíster en Educación: Desarrollo Humano, Especialista en Docencia Universitaria, Terapeuta Ocupacional. Profesora Asistente Universidad del Valle. Facultad de Salud. Escuela de Rehabilitación Humana. Programa Académico de Terapia Ocupacional. Cali (Colombia). Contacto: aida.rojas@,correounivalle.edu.co 


\section{Introducción.}

En la actualidad, en las Universidades de Colombia la evaluación profesoral se identifica como una actividad sumatoria, en donde se tiene en cuenta principalmente el criterio de los resultados de "desempeño docente", el cual es valorado por los estudiantes, lo cual se compara con algunos logros que institucionalmente se desean lograr.

Este enfoque se ha cuestionado debido a su corte instrumental, con énfasis técnico, el cual es insuficiente para mejorar el desempeño profesoral. De igual manera no considera el proceso formativo del profesor, su aspecto personal, ni su impacto en la formación de los estudiantes.

La llamada evaluación del desempeño docente es usualmente considerada como un proceso que hace seguimiento al quehacer del profesor en su labor con los estudiantes, es decir, se centra en su función "docente", que genera información sobre todo lo realizado desde el inicio hasta el final de cada periodo académico, pero en realidad el objetivo no se alcanza, pues muchas de estas evaluaciones se realizan solo al finalizar la asignatura y no tienen parámetros que retroalimenten al profesor o verifiquen su mejoramiento.

Por esto resulta importante emprender nuevas miradas e iniciativas de la evaluación que se planteen desde los sentidos que los mismos profesores universitarios le confieren al concepto de evaluación. Re-conceptualizar y re-configurar la evaluación profesoral, requiere que se construya, desde los puntos de encuentro o desencuentro de los sentidos que los profesores le atribuyen a la evaluación en su relación con la práctica pedagógica, la pertinencia social y las políticas de calidad institucional.

Surge como respuesta a estos sentidos referidos por los mismos profesores, la evaluación dialogante, la cual es una propuesta de orden epistemológico, que busca aportar a la evaluación profesoral, en donde se propicie una cultura evaluativa, pero que a la vez aporte de manera mucho más asertiva a los procesos formativos.

En el artículo se presenta dicha propuesta epistemológica, en donde se plantean los fundamentos empíricos, teóricos, pedagógicos, epistemológicos, ontológicos y éticos de la evaluación dialogante.

La evaluación dialogante es ante todo una propuesta epistemológica, resultado de una investigación que comprendió los sentidos de la evaluación profesoral en la Educación Superior. Dicha evaluación contribuye a afianzar el enfoque dialogante, tanto de la formación como de la evaluación, más que propiamente un método evaluativo aislado.

\section{Fundamentos empíricos de la evaluación dialogante.}

Los sentidos que le otorgan los profesores de Terapia Ocupacional a la evaluación profesoral, se esquematizan a continuación: 
Ilustración 1. Resultados sentidos otorgados a la evaluación profesoral.

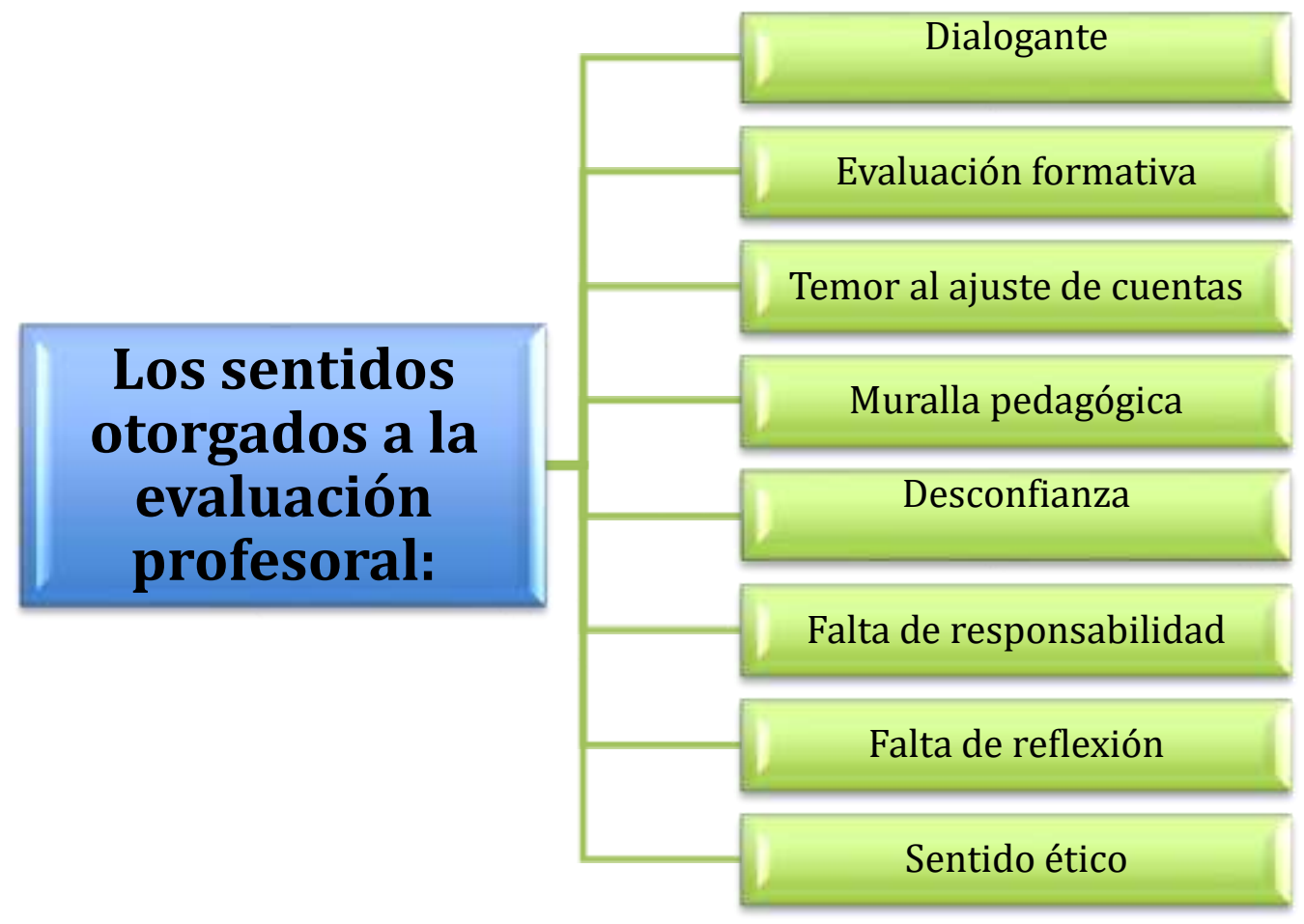

Fuente: Elaboración propia.

La evaluación dialogante es para docentes, estudiantes y egresados, la que denota más sentido en la práctica pedagógica frente al sentido que revela la evaluación profesoral formal. Esta forma de concebir la evaluación no ha sido reconocida ni aplicada (a la fecha de la investigación), para valorar el quehacer de los profesores de los programas de Terapia Ocupacional en Colombia (Universidad del Rosario y Universidad del Valle).

Este tipo de evaluación constituye un hallazgo innovador, puesto que a partir de ahí se origina la construcción conceptual del enfoque que ahora se propone. Nos encontramos frente a unos sentidos que plantean una nueva perspectiva de la evaluación del profesor en educación superior, la evaluación dialogante, ante lo cual surgen una serie de preguntas:

¿Pueden y deben los profesores reconocer el diálogo evaluativo con los estudiantes como un enfoque capaz de mejorar la práctica pedagógica, e incluso capaz de transformar el currículo? Si así fuera, ¿qué principios ontológicos, epistémicos y metodológicos sustentarían al diálogo como un enfoque evaluativo innovador y diferente? En resumen, ¿qué implicaciones tiene una evaluación dialogante para la formación de los profesionales y para el proceso de evaluación de los profesores universitarios de los programas de Terapia Ocupacional?

\section{Fundamentos teóricos de la evaluación dialogante.}

La creación del conocimiento se realiza dentro y fuera del salón de clase, por tal razón una función de las instituciones educativas es de formadora de cultura humana. Es por esto que se privilegian y defienden las acciones y el rol del profesor como componentes centrales en todo proceso educativo. 
La relación pedagógica está mediada por relaciones sociales, culturales y políticas, en ocasiones complejas. En donde la intención del contexto histórico, lo socio-cultural y lo político, son núcleos que logran definir la práctica educativa como tal.

El profesor universitario, que, para efectos de la investigación, es el Terapeuta Ocupacional que ejecuta acciones de formación disciplinar, el cual debe poseer habilidades y destrezas propias de la profesión, pero además, debe tener la capacidad de realizar actividades propias de la práctica pedagógica y estar inmerso dentro de la estructura curricular de la institución correspondiente.

Las diferentes experiencias personales, académicas y profesionales ayudan a forjar un camino dentro del quehacer profesoral, cada profesor de Terapia Ocupacional tiene sus características las cuales le permiten valorar el ritmo y estilo de aprendizaje de cada estudiante, es importante resarcir el buen trato, la interlocución y propender por que exista un ambiente agradable de trabajo.

Se consideran cuatro elementos claves en la conceptualización del profesor universitario de Terapia Ocupacional dentro de la investigación, que se relacionan con, la parte humana, lo que se requiere para ser profesor universitario, la relación estudiante - profesor y el papel del profesor universitario en la sociedad.

Desafortunadamente en los espacios académicos se ha dejado de lado el componente afectivo y se le da mayor importancia a lo cognitivo; se nos olvida que el ser humano logra aprender fácilmente cuando está de por medio lo afectivo. Pues si la autoestima está bien estructurada, se facilitan los procesos de aprendizaje. Los aprendizajes significativos están mediados por el amor y el ambiente agradable de trabajo.

Los procesos de formación deben hacerse con entusiasmo, motivación, se debe dejar de lado la rutina y lo repetitivo, para poder acercarnos de manera más fácil y cordial a los estudiantes. En el proceso de enseñanza y aprendizaje es importante resaltar que debe existir un trabajo conjunto, pero sobre todo que se debe orientar al estudiante hacia un aprendizaje crítico.

El profesor juega un papel estratégico en las transformaciones educativas, puesto que en el aula de clase se medían relaciones que influyen en el desarrollo profesional, personal e institucional.

En la relación estudiante - profesor, se debe tener en cuenta que no solo es importante hablar, sino que también se debe saber escuchar. Lo cual es básico para poder crear un verdadero diálogo y unos vínculos de confianza, que permitan acercamiento muto.

En la medida que haya más diálogo, se van a acortar las distancias, lo cual mediará de manera favorable en la interacción estudiante - profesor.

El reto que se tiene como profesores universitarios, es generar una educación que se convierta en un estilo de vida, que abarque todos los instantes. En una forma de interiorizar una manera de vivir acorde con lo natural, en un estado de ánimo, una disposición, una filosofía de vida, en un intento por estar despierto para ver y percibir, en una construcción y edificación personal de las experiencias afectivas sanas. 
El tema de sentido, tiene importancia y se relaciona con la evaluación profesoral, pues los dispensadores de sentido, así como la posibilidad sostenida en el tiempo le otorgan al profesor de Terapia Ocupacional razones suficientes para realizar cambios en sus prácticas, que le ayudan a definir el porqué de su quehacer y así lograr entender y por lo tanto ejecutar sus funciones generando cambios favorables dentro de lo instaurado por el Estado y la Institución en donde se encuentra.

\section{Fundamentos pedagógicos de la evaluación dialogante.}

La evaluación dialogante facilita el encuentro profesor-estudiante, como un espacio recíproco que activa el proceso educativo y dinamiza la práctica pedagógica.

En primer lugar, es importante el encuentro cara a cara, porque facilita la comunicación, la interacción, y enriquece el diálogo de manera recíproca, tal como lo propone Paulo Freire (2002), el estudiante ya no es un objeto vacío para llenar, sino que se convierte en un sujeto activo y transformador de práctica. Una educación que reconoce al otro como sujeto responsable y actor de su propio destino, se fundamenta en el respeto por el otro y en la práctica de relaciones horizontales, pero parte de reconocer que los seres humanos están inconclusos y se construyen en la relación con el otro y con el mundo. Es, por tanto, una relación que se nutre de amor, humildad, esperanza, fe y confianza en el otro, donde ambos se hacen críticos en la búsqueda de algo y crean una relación de simpatía; cada persona llega a ser ella misma sólo cuando los demás también lleguen a ser ellos mismos.

La evaluación dialogante de acuerdo con Fernández (1999) reconoce al profesor y al estudiante como seres de relaciones humanas, de naturaleza plural, crítica, trascendente, temporal y creadora, gracias a la cual el momento evaluativo permite y da apertura a espacios de confianza, respeto y participación, los cuales permiten intercambio de miradas, información, reflexiones, análisis, que sugieran cambios desde lo académico, pedagógico, evaluativo y curricular. Es un espacio recíproco porque propicia el crecimiento conjunto de profesores y estudiantes, en la medida que el profesor re-conoce y re-flexiona sobre su quehacer, sus prácticas, el currículo, y los estudiantes; de manera simultánea, se verán motivados a cuestionar y replantear su experiencia educativa, que permita orientar y facilitar su aprendizaje, para su crecimiento personal y futuro desempeño profesional.

El análisis y la reflexión llevan a los actores comprometidos, a relacionar los resultados, con las expectativas y objetivos iniciales, y de esta manera, poder conocer el impacto de lo que se ha desarrollado (Rueda \& Díaz, 2011)

La re-alimentación del currículo se logra cuando, tanto profesores como estudiantes asimilan sus debilidades y fortalezas, lo cual se consigue en la medida que se genera una verdadera cultura evaluativa. De esta manera se logra interiorizar la importancia de la evaluación, aspecto que favorece lo académico, pero también propicia el avance en la construcción de sujetos, pues el diálogo no se centra solamente en el aprendizaje según Julián de Zubiría (2006), por esto la importancia de facilitar espacios abiertos, reflexivos y que fomenten la participación.

La evaluación dialogante posibilita la re-activación del proceso educativo, porque precisamente es a partir de este trabajo conjunto y responsable, que se permite la movilización de las prácticas pedagógicas y los procesos curriculares del programa. 
Por ser una tarea con responsabilidad compartida, se hace conveniente crear una verdadera cultura de la evaluación, donde primen las creencias y las convicciones de la comunidad educativa, frente a la enseñanza y el aprendizaje.

Es imperativo luchar contra lo mecánico, contra lo rutinario y convencional. Se activará el proceso educativo, para ir en contravía de la inercia educativa (De Zubiría, 2006)este es el aporte de la reflexión metodológica, enmarcada dentro de la reflexión curricular.

Lo anterior, permite que la práctica pedagógica se dinamice, pues, a través de la evaluación dialogante, se realizará un trabajo conjunto, que permite la reflexión y el análisis. Por lo tanto, la práctica pedagógica se ve estimulada, a partir de este proceso conjunto que se lleva a cabo entre profesores y estudiantes.

La evaluación dialogante, además, es una forma de mirar al interior y de esta manera identificar qué tanta mejora o mantienen sus prácticas los profesores. Esto, apoyado en el concepto de evaluación responsiva la cual de acuerdo con Scriven (1967), considera la evaluación como elemento de información continua, la cual es fundamental para planificar y luego poder ver resultados de los procedimientos que han sido utilizados por los profesores, para luego acondicionar las prácticas pedagógicas de acuerdo a las necesidades que se han detectado en sus estudiantes.

Así mismo facilita el encuentro profesor-estudiante, como espacio recíproco que activa el proceso educativo y dinamiza la práctica pedagógica, tiene unas implicaciones en el plano educativo que son fundamentales, porque llevarán de la mano lo curricular con la evaluación (Santos, 1993) de no verse como algo añadido al final, sino como parte de un proceso, de ese todo que es lo académico - curricular.

Para cumplir con el propósito de encuentro profesor-estudiante, los participantes serán valorados y respetados, respetuosos y responsables, siguiendo de esta manera la idea de diálogo como una pedagogía igualitaria (Burbules \& Bruce, 2001).

La evaluación dialogante busca la objetividad, el análisis, la reflexión, la re-alimentación. Para que esta evaluación cumpla con su objetivo: ser formativa, se hace necesario discutir la polaridad subjetiva, objetiva e intersubjetiva, que vendría a corresponder precisamente al diálogo.

De igual manera la evaluación dialogante favorece las actitudes abiertas, dinámicas, crea experiencias, sensibles, responsables y respetuosas entre profesor-estudiante, profesor- profesor y profesor-directivo.

Así mismo, contribuye a la formación profesional del profesor, porque los procesos evaluativos se vinculan con las estrategias institucionales de formación docente para contribuir en la profesionalización de los profesores, de acuerdo con Rueda, M. y Díaz, M. (2001). Pero todos estos procesos cuentan con la apertura y actitud de responsabilidad, respeto y honestidad de cada profesor, y de cada estudiante, pues esto no funciona como un lineamiento unidireccional, es algo que nace y se promueve desde cada uno. 
En la medida que la comunidad académica sea sensible, desarrolle inteligencia emocional, reconozca y comunique su situación emocional, así como que sea sensible a la emocionalidad del otro, se favorecerá el dialogo. Desde la biología del amor de la cual habla Humberto Maturana (2001), se explica precisamente la importancia de este aspecto, pues educar es un fenómeno biológico que involucra todas las dimensiones del ser humano, que integra los aspectos corporal, emocional, social y espiritual; la educación es un proceso de transformación en la convivencia, donde el sujeto cambia junto su comunidad, gracias a que puede relacionar el carácter dinámico del lenguaje, compartir e interactuar en el amor. El dialogo implica y exige conciencia de sí mismo, del otro y del grupo social, respeto y responsabilidad por sí mismo y por los otros. El amor más que un sentimiento, es la esencia de nuestra vida y el soporte de la convivencia (Maturana, 1997).

La inteligencia emocional, por su parte (Goleman, 1996), hace que como seres humanos se tome conciencia de las propias emociones, que se entiendan los sentimientos de los otros, de igual manera hace entender la forma como se logran tolerar las tensiones y frustraciones que se presentan en el día a día y fortalece la capacidad de trabajo en equipo, lo cual amplia la perspectiva de desarrollo personal.

Por tanto, en la medida que los espacios educativos favorezcan la acción y la reflexión, sobre las emociones, pensamientos, deseos, opiniones y actos, se hace un acto de desapego (Maturana, 1997), el cual mejora la relación consigo mismo, y la relación con los otros, porque en la medida que hay sensibilidad, honestidad y respeto con uno mismo, se logra escuchar al otro.

El trabajo académico es una responsabilidad compartida, la cual se debe dinamizar. El compromiso es de todos: profesores, estudiantes y directivos; en la medida que el diálogo y la reflexión fluyan entre todos, se le estará dando entrada directa a la cultura evaluativa, lo cual terminará con el fantasma punitivo que ronda la evaluación. Esto no será tan fácil, lo importante es que surja un cambio de actitud recíproco ante los demás, que abra las puertas del cambio.

El diálogo favorece las relaciones horizontales, y aquí se incluyen todas aquellas que median la academia: profesor - estudiante, profesor - profesor y profesor - directivo, como pilares de formación. Se parte de que dicha relación se basa en la confianza. En La pedagogía del oprimido de Paulo Freire (1970), se asegura que el diálogo basado en el amor, la humildad y la fe llevan a la confianza. Estos elementos resultan claves en los procesos formativos, pues decir una cosa y hacer otra, desestimula y rompe la credibilidad.

Una ventaja que ofrece la evaluación dialogante es la autonomía. Al permitir encuentros profesor-estudiante, profesor-profesor y profesor-directivo, cada uno es responsable de su palabra, de su acción; asume sus consecuencias y sus desafíos, dinamizando así el aprendizaje y la enseñanza.

La evaluación dialogante guía hacia unos procesos académicos, que incluyen el respeto y la confianza, lo cual es fundamental dentro de lo educativo, pues todo ser humano necesita ser respetado, con sus individualidades y características. Esos lazos de respeto y confianza facilitan los vínculos afectivos que apoyan relaciones cordiales, las cuales posibilitan el acercamiento entre los miembros de la comunidad académica, y máximo cuando las emociones son consideradas como dinámicas corporales que ayudan a especificar el dominio de su actuar, dentro del contexto en el que se mueve cada individuo (Maturana, 1997). 


\section{Fundamentos epistemológicos de la evaluación dialogante.}

La concepción actual de la evaluación se aleja de lo tradicional, que la veía como algo que calificaba, sancionaba, valoraba y media según Díaz (2005). Actualmente, la evaluación le apunta a una intervención conjunta, que parte de una información inicial, no solo final; que apela a un proceso de indagación crítico y sistemático, en la que se hacen juicios de valor sobre las personas, las situaciones, un fenómeno, dicho juicio se hace sobre unos criterios establecidos de manera previa, su objetivo principal es la toma de decisiones, a su vez, estas decisiones son orientadas hacia el mejoramiento de la enseñanza, lo que permite una comunicación directa entre profesores y estudiantes.

No obstante, lo anterior, vale la pena resaltar que dicha comunicación no necesariamente implica una educación y evaluación dialógica, pues si no existe un contexto académico y condiciones institucionales que favorezcan y permitan el diálogo, no se cumplirá este propósito. El profesor, además, debe estar convencido de la importancia de este tipo de trabajo dialógico, pues puede - saber sobre el diálogo, pero no colocarlo en práctica (Velasco \& Alonso, 2008).

La evaluación a través del tiempo ha tenido varias modificaciones conceptuales, por las diferentes construcciones que han surgido sobre ella, aun así, es fuerte su contribución en la calificación de niveles de aprendizaje y promoción de estudiantes (Pérez, 2005). Hasta ahora ha sido escasa la atención puesta por los pensadores y teóricos de la evaluación, en la evaluación de los profesores. La evaluación de los profesores constituye un elemento esencial del proceso educativo y un pilar del ethos académico, que se verá reflejada en la formación de los profesionales. Recordando el viejo adagio - con la vara que midas serás medido -, la evaluación del profesor constituye un proceso que marca un horizonte, genera significados profundos y determina un valor cultural, que tendrá influencia recíproca en la vida de estudiantes y profesores.

La mirada controladora, fiscalizadora y medidora, que instrumentaliza, reduce y cosifica al sujeto ignora y menoscaba la confianza responsabilidad y autonomía de quienes son evaluados. Ante un evento tan importante como es la evaluación del profesor, deben ser reconocidos explícitamente no solo las capacidades y actividades pedagógicas, investigativas y profesionales del maestro, sino además sus comportamientos, relaciones y valores humanos, porque la evaluación debe incluir al sujeto educador integralmente en todas sus dimensiones, si se aspira que este evalúe integralmente las dimensiones del sujeto educado.

Por lo anterior, resulta fundamental salir del dominio normativo de la evaluación, que la encasilla en algo sumativo y de medición, es decir, darle apertura a la dimensión formativa, que lleve, tanto a profesores como a estudiantes, a explorar nuevas formas de relación, crecimiento y aprendizaje.

\section{Fundamentos ontológicos de la evaluación dialogante.}

La evaluación dialogante es un camino para estimular y desarrollar habilidades reflexivas y de razonamiento en la comunidad académica. 
Para lograr el encuentro, la interacción y facilitar una mentalidad abierta, es importante contar con habilidades sociales, cognitivas y comunicativas, estas habilidades no necesariamente son innatas, pueden desarrollarse a través del tiempo y la experiencia.

La evaluación dialogante demanda ciertas habilidades desde la reflexión, para que tanto estudiantes como profesores tengan clara la diferencia entre la crítica constructiva y la que no lo es tanto, así como la posibilidad de entender que lo puesto sobre la mesa no es algo personal.

El diálogo crítico-reflexivo es un camino que se utiliza en los procesos de enseñanza y aprendizaje, apoyando el desarrollo del ser humano. Julián de Zubiría (2006) afirma que la finalidad del trabajo, basado en el diálogo, debe mejorar los niveles de pensamiento, afecto y acción. Es por esto que la evaluación basada sólo en lo instrumental, no facilita la reflexión, lo que no permite el mejoramiento continuo del proceso de enseñanza-aprendizaje.

A través de un diálogo concertado y reflexivo se logran mejores resultados, que cuando se hace un trabajo obligado y descontextualizado. En el aula se espera poder compartir experiencias, es decir, que el profesor entrega lo mejor de sí, pero de igual manera espera recibir. En este sentido, las clases operan como un escenario de crecimiento mutuo para estudiantes y profesores, por esto las experiencias sumativas no cumplen con objetivos pedagógicos, pues se vuelven sólo rendición de cuentas, sin aportar al crecimiento conjunto (Rueda \& Díaz, 2011).

El encuentro cara a cara es fundamental para poder estimular la reflexión y el razonamiento. Son las discusiones en el aula las que generan la reflexión, a través del diálogo, y van ofreciendo experiencia, para adquirir habilidades reflexivas y de razonamiento, necesarias para una discusión rica en argumentos.

Cuando la evaluación dialogante permita que el profesor hable con sí mismo, que haga su reflexión y análisis, se logrará que haga una reelaboración de su práctica pedagógica, que asimile lo dicho de manera individualizada, es decir, logrará apropiarse de su vivencia evaluativa.

Not (1992) por su parte considera que la cultura evaluativa dependerá de la manera en que tanto estudiantes como profesores, integren la actividad evaluativa a su discurso diario .

La evaluación dialogante también es una forma de participación que torna co- responsables a profesores, estudiantes y directivos en el proceso evaluativo.

La evaluación actual reclama que los estudiantes sean proactivos en el momento de su realización, que participen de manera más activa, que no sean solamente observadores de un proceso en el que ellos tienen mucha injerencia.

Dicho proceso evaluativo debe enriquecer los procesos académicos, pedagógicos y curriculares, por esto es importante saber aprovecharla, y como tiene varios aristas, son los mismos participantes quienes pueden visualizarlas, pero este trabajo debe ser consciente, responsable y dinámico, para que la reflexión aporte en la educación. El objetivo de un trabajo dialogante debe ser el desarrollo y no sólo el aprendizaje (De Zubiría, 2006).

Lo opuesto al diálogo, el silencio puede convertirse en cómplice de un proceso educativo mal realizado, por esto es importante que, de manera responsable, los diferentes actores participen y 
analicen lo que sucede en los espacios académicos. Cuando se elabora un trabajo responsable y concienzudo, independiente de cómo se esté llevando a cabo la tarea, siempre habrá elementos para elaborar una retroalimentación.

La responsabilidad frente a los procesos evaluativos para los estudiantes, se va haciendo más consciente en la medida en que aumentan los semestres académicos, pues van teniendo más claridad sobre lo que es su carrera, pero igualmente toman consciencia frente al proceso evaluativo, entendiendo que no es un proceso que les corresponde sólo a ellos de manera individual, sino que tienen una - responsabilidad social - frente a los compañeros de otros semestres. El análisis y la reflexión aportan, en este sentido, más que la sola calificación, el cuestionario sólo le da una mirada al profesor de transmisor de información y no toda la connotación que en realidad la evaluación tiene (Rueda, M. \& Díaz, F. 2011).

Humberto Maturana (2002) considera que este compromiso y responsabilidad, de dinamizar la evaluación profesoral es, de manera conjunta, de profesores, estudiantes y directivos, por tanto, debe ser un acto consciente, que aporte al mejoramiento de las prácticas pedagógicas, pero así mismo que contribuya en la construcción de mejores seres humanos, porque en la educación es donde se da la transformación de la convivencia, lo humano y el ser humano.

La evaluación contribuye a la construcción de sujetos, por esto, pensando en ese ser humano con el cual se está trabajando, es que se deben resaltar tanto los puntos positivos, como los negativos, pues ellos ayudarán a sacar un mejor provecho de este proceso evaluativo, porque aprenderán de sus errores, pero también podrán potencializar lo que han venido haciendo bien. Puesto que la evaluación profesoral no debe ser una amenaza, al contrario, debe permitir la relación entre seres humanos que permita su cambio (Maturana, 2002).

La evaluación dialogante permitirá hacer de la evaluación profesoral algo consciente, con un claro compromiso y con un sentido de responsabilidad, evitando que el objetivo sea dejar al profesor en evidencia frente a la comunidad académica. De igual manera, evitará la comparación y dejar en desventaja a aquellos profesores que fueron mal evaluados.

El profesor ante todo es un ser humano, una persona, de esta manera, uno de los fundamentos de la evaluación dialogante es preservar la dignidad, autoestima e individualidad de los profesores (Rueda \& Díaz, 2011).

El diálogo llevará a realizar auto-reflexiones y reflexiones sobre el quehacer del profesor, que serán animadas por el diálogo con los estudiantes, aspecto importante para lograr recuperar las dimensiones humanas, lo que le permitirá al profesor vivir y mirar la realidad de una manera diferente, descubriéndose a sí mismo y legitimando al otro (Maturana, 2002), lo cual servirá como orientador para identificar su responsabilidad dentro del proceso evaluativo.

\section{Fundamentos éticos de la evaluación dialogante.}

En su dimensión ética, la evaluación dialogante fomenta la autonomía y la justicia en el proceso educativo.

La evaluación dialogante le apunta a la ética, con unos principios que buscan la autonomía de la comunidad académica; al tiempo que procura el logro de beneficios, reduciendo el riesgo de 
daños e injusticias; de igual forma, busca que los aspectos que se tienen como diferentes sean atendidos, de tal manera que la diferencia sea reconocida. Siguiendo estos principios, la evaluación, busca hacer un reconocimiento por igual de todos los participantes de la comunidad académica, sin marcar diferencias entre buenos y malos, simplemente potenciando escenarios de diálogo.

Las clases son espacios de aprendizaje, en donde los estudiantes podrán preguntar libremente, para aclarar sus dudas; de igual manera, el profesor podrá sentirse libre de expresar sus ideas y conocimientos; pero en caso de no saber algo, también está en la libertad de expresar que lo va a consultar para poder exponerlo de mejor manera. La ética de la práctica educativa de acuerdo con Paulo Freire (2002) debe vivirse en el hacer diario, cuando enseñamos, citamos autores, preguntamos, despejamos dudas.

La preparación científica del profesor debe ser coherente con sus aspectos éticos. Es fundamental aprender de la diferencia, por esto no se le debe dar cabida a asuntos de tipo personal o a la antipatía hacia el otro, en el momento de analizar el cumplimiento de las obligaciones y conceptos (Freire, 2002).

Es importante el seguimiento que se le haga a la evaluación, no necesariamente por terceros, sino por cada uno de los actores participantes, permitiendo la revisión del plan de trabajo, así como un análisis sobre los resultados obtenidos, elementos que le darán claridad al docente frente a cómo está realizando el trabajo y cómo está siendo visto por la comunidad académica. Siempre habrá diferencias en las evaluaciones realizadas y dichas posiciones antagónicas son importantes para poder clarificar lo que sucede en el aula de clase, por tal razón deben aceptarse con respeto y lealtad (Freire, 2002).

La evaluación dialogante permitirá que haya un trabajo conjunto, evitará que el profesor se tenga que salir del salón en el momento en que se vaya a realizar la actividad evaluativa, que sea rezagado de su propio proceso evaluativo; al contrario, lo hará participe, le dará voz. Mélich \& Boixader (2011) consideran que la ética es la esencia de la relación educativa, por esto la importancia de realizar este trabajo conjunto.

El principio de justicia en la evaluación dialogante, permitirá crear estrategias que lleven a desarrollar capacidades, de acuerdo con intereses y necesidades de la población, que permitirá potenciarlas (Murillo, et al, 2011). Buscando disminuir los temores tanto de profesores como de estudiantes, a través de una reflexión conjunta, que evitará que se tome la evaluación como un mecanismo de venganza o de revancha.

Pero se considera que la evaluación, pese a manejar estándares altos de rigurosidad y pertinencia, puede perder su sentido de acuerdo con el uso que de ella se haga (Murillo, et al, 2011). Alberto Martínez (2004) nos coloca de ejemplo, la entrada de la administración a la educación, lo cual pervirtió elementos esenciales de la evaluación, porque se le ha dado un valor salarial, se maneja como un producto, como una mercancía, no se le da el cultivo del espíritu humano, de la libertad, de la razón, es decir, los maestros se mueven con base en necesidades laborales o de los indicadores de calidad (Martínez, 2004). Por esto, la relevancia de trabajar la evaluación a través del análisis y la reflexión, la cual no debe estar alejada del respeto, la responsabilidad, el compromiso y el sentido de pertenencia.

\begin{tabular}{l|l|l|l|l|l|l} 
AGO.USB & Medellín - Colombia & Vol. 17 No. 1 & PP 1 - 323 & enero - junio & 2017 & ISSN: 16578031
\end{tabular} 
La ética está inmersa en todas las vivencias de la vida de uno, en cada acto se muestra un comportamiento moral, entonces la ética, no tiene que ver ni con los espacios públicos, ni con los espacios privados, sino en revisar, reflexionar por qué los comportamientos morales de las personas son así. Se logra una educación justa y que vaya de acuerdo con la justicia social, si se cuenta con una evaluación justa, inclusiva, equitativa, participativa, democrática, no represiva, cuyo fin último sea la reflexión y el mejoramiento continuo (Murillo, et al, 2011).

El diálogo contribuye a realizar esa reflexión, que finalmente lleva a actitudes éticas, puesto que el carácter social de la ética depende del amor y no de la razón. En la medida en que el fenómeno del amor esté fundamentado en lo biológico de lo humano, de acuerdo con Humberto Maturana (1997) la ética estará siempre presente. Pues cuando el ser humano se ama, lo que desea y busca para él mismo es la excelencia, por esto es imposible que una persona que mantenga este equilibrio, vaya a buscar la destrucción o ir contra de lo que el entorno le exige (Universidad de Antioquia, sf).

La ética es la esencia de la educación, según lo referido por Mélich y Boixader (2011) porque la ética es la naturaleza de la formación, de allí que todo ejercicio de evaluación retorna a la formación.

La evaluación dialogante llevará a reflexionar sobre lo que se hace en la práctica educativa, lo cual tiene implicaciones éticas y morales. Aporta en los niveles de inclusión en la educación y permite construir diversidad de estrategias que se verán reflejados en las habilidades adquiridas por la comunidad académica (Murillo, et al, 2011). Aspectos que se transforman en ética, al reflexionar sobre la manera cómo se comporta el individuo y la forma cómo hace las cosas.

La enseñanza eficaz se logra cuando hay un orden moral básico, puesto que la educación implica una mejora de los demás, en el sentido de la formación personal, que trascenderá a un solo entrenamiento o instrucción (Carr, 2005).

La evaluación dialogante permite un verdadero ejercicio de reflexión-acción, para que los comportamientos se modifiquen, porque se han interiorizado y no por miedo a la sanción, pues la ética marca la naturaleza humana. La ética es necesaria para facilitar la convivencia de los seres humanos (Freire, 2002).

\section{Conclusiones.}

El trabajar la evaluación profesoral desde el diálogo, posibilita la escucha, la expresión franca, despojada de ataduras, facilita los elementos de control y poder que se manejan en los espacios académicos, los cuales podrán ser asumidos por ambos protagonistas de manera responsable y recíproca. Esto porque tanto el profesor como los estudiantes entran en una igualdad de condiciones en ese espacio de encuentro, razón por la cual puede llegar a fluir el diálogo como un proceso bilateral, democrático y plural de la actividad evaluativa. Lo que lleva a que se diluya la autoridad unilateral del profesor y aún de los estudiantes como evaluadores de unos a otros, pasando a hacerlo en conjunto.

El diálogo como elemento social, en donde está inmerso el fenómeno humano, está mediado por la palabra, es decir que el diálogo es un espacio para la palabra. 
La palabra es un elemento fundamental para la comunicación y el conocimiento, dicha palabra tiene sentido si está mediada por la reflexión, así se logrará algo verdaderamente transformador. De lo contrario sería una acción sin objetivo, se limitaría a palabrerías. El ser humano crece por el diálogo reflexivo.

El diálogo como espacio reflexivo en la evaluación, no está basado en cualquier tipo de conversación, porque lo que se busca es producir conocimiento, el cual se consigue cuando hay diálogo interno, diálogo con el otro y diálogo con el ser. El diálogo de por sí tiene la finalidad del entendimiento, el acuerdo y la coordinación de acciones (Habermas, 1981). Sócrates alentó el dialogar. Fue su método para contribuir a que su interlocutor alcanzara la sabiduría (Ibarra, Rodríguez, \& Gómez, 2012).

El diálogo, el conocimiento y la educación conforman una triada que orienta decisiones de los profesores tendientes a propiciar o dirigir a aprender, conocer, pensar, actuar inteligentemente, reflexionar o, bien, a jugar y perder el tiempo en un delicioso y divertido ocio (Ibarra L. , 2010). Por esto es fundamental que el profesor entre en diálogo con los estudiantes, en donde se tenga en cuenta la educación centrada en el aprendizaje, los estudiantes y la manera impulsar el aprendizaje, el conocimiento, el pensamiento, la actuación con relación a la inteligencia y la reflexión.

Lo que se busca con el enfoque de evaluación dialogante es algo más que una simple conversación, pues debe llevar a la reflexión y a descubrir al propio ser, en donde lo que se busca es conseguir mejores preguntas, puesto que el objetivo no es llegar a la total claridad, sino poder identificar en cuáles aspectos hay obscuridad, para poder identificar de esta manera que es lo que no se entiende, en dónde faltan acuerdos, y cuáles son las diferentes necesidades que hay en la comunidad académica participante.

El diálogo se presenta como espacio democrático, en donde se piensa en la humanización de la educación, se procura en condiciones que garanticen la libertad de opinión y por lo tanto que la información obtenida sea tenida en cuenta y se utilice de manera apropiada. Este tipo de trabajo facilita la concepción democrática, lo cual permitirá mejores acercamientos, mayor confianza, y por lo tanto apertura sobre las diversas opiniones que surjan.

El diálogo enriquece los procesos educativos, en donde se incluye la evaluación profesoral, si se lleva a cabo en términos de libertad y si hay una verdadera actitud de apertura. Este diálogo debe ser de calidad y cada participante tendrá su momento de protagonismo.

El diálogo que propicia aprendizaje, es de por sí, ético, porque crea nuevos espacios de diálogo; esto es, el diálogo es circular. Es un procedimiento democrático y un conjunto de habilidades de interrelación, que permiten interrelacionar diversas modalidades de aprendizaje basadas en experiencias de diálogo y modalidades de aprendizaje, las cuales se orientan a la conceptualización, pero además aportan a crear nuevos mecanismos de diálogo (Puig, J. 2007).

Dicho diálogo será un espacio de construcción pedagógico, será un trabajo humano, en busca de resultados más fructíferos, debido a que los profesores y estudiantes construyen espacios o momento de desconfianza que pueden sostenerse en experiencias previas con otros actores o surgir de circunstancias al interior del acto educativo. Sin embargo, en la medida que el poder y la jerarquización queden a un lado y se faciliten acuerdos basados en la reflexión, el pensamiento, 
el aprendizaje y el conocimiento ya bien sea individual o colectivo, se derrumbará la muralla de la desconfianza. De esta manera también se evitará la imposición y el engaño.

El diálogo como constructor de sentidos y significados, permitirá a profesores y estudiantes lograr una orientación, una justificación y un vínculo con lo que hacen, de esta manera se incidirá en el crecimiento mutuo, en donde tanto los profesores como los estudiantes le den un lugar especial a escuchar, atender y comprender, aspectos que se presenten y discutan en el plano académico y afectivo dentro de un contexto educativo.

El ser humano logra humanizarse gracias a los procesos educativos. Por esto la importancia del diálogo en la evaluación, pues contribuye a este proceso humanizante en la educación. El diálogo construye humanidad, porque es en diálogo que las personas aprenden a ser libres, autónomas, reflexivas, analíticas, independiente, por tanto, el diálogo es formativo.

La evaluación profesoral debe proporcionar información que permita ser un punto de partida para tomar decisiones frente a los elementos que hacen parte del proceso educativo. Por esto debe estar al servicio de la comprensión y por tanto de la formación.

Se estima que el poder y el autoritarismo excluyen al diálogo, pues se parte de un elemento en donde se considera que la única verdad es la de cada agente participante y se desacredita lo que los otros dicen, es un esfuerzo por fortificar el dominio. Por esto se corrobora que el diálogo genuino en la evaluación profesoral, aquel que busca la verdad y desea fomentar el conocimiento sin estar mediado por prejuicios aporta al mejoramiento de la praxis profesoral y del currículo en el Programa Académico de Terapia Ocupacional. Así como favorece relaciones de confianza, dejando de lado el poder que impone el proceso evaluativo.

\section{Bibliografía.}

Burbules, N., \& Bruce, B. (2001). Teoría e Investigación en Enseñanza como Diálogo. Washington D.C: American Educational Research Association - Universidad de Illinois.

Carr, D. (2005). El Sentido de la Educación. Una introducción a la filosofía y a la teoría de la educación y de la enseñanza. Barcelona: Editorial Graró.

De Zubiría, J. (2006). Los Modelos Pedagógicos. Hacia una pedagogía dialogante. Bogotá: Editorial Aula Abierta Magisterio.

Díaz, J. (2005). La Evaluación Formativa como Instrumento de Aprendizaje en Educación Física. Zaragoza: Publicaciones Inde.

Fernández, J. M. (1999). Paulo Freire: Una propuesta de comunicación para la educación en América Latina. Revista Razón y Palabra(13). Obtenido de http://www.razonypalabra.org.mx/anteriores/n13/freirem13.html

Freire, P. (1970). Pedagogía del Oprimido. México: Siglo XXI Editores.

Freire, P. (2002). Pedagogía de la Autonomía. Saberes necesarios para la práctica educativa. México D.F.:

Siglo XXI Editores. 
Goleman, D. (1996). Inteligencia Emocional. Barcelona: Editorial Kairós.

Habermas, J. (1981). Teoría de la acción comunicativa. Madrid: Taurus Ediciones.

Ibarra, L. (2010). Sabiduría: Diálogo y Educación. Actualidades Investigativas en Educación, 10(2), 1-33. Obtenido de http://revistas.ucr.ac.cr/index.php/aie/article/view/10110

Ibarra, S., Rodríguez, G., \& Gómez, M. (2012). La evaluación entre iguales: beneficios y estrategias para su práctica en la universidad. Revista de Educación(359), 206-231. Obtenido de http://www.mecd.gob.es/dctm/revista-deeducacion/articulosre359/re35911.pdf?documentId=0901e72b813d72cf

Martínez, A. (2004). De la Escuela Expansiva a la Escuela Competitiva. Barcelona: Editorial Artrópodos.

Maturana, H. (1997). Emociones y Lenguaje en Educación y Política. Santiago de Chile: J.C. Sáez. Maturana, H. (2002). Formación Humana y Capacitación. Santiago de Chile: Dolmen Ediciones.

Mélich, J. C., \& Boixader, A. (2011). Los Márgenes de la Moral. Una mirada ética a la Educación. Revista Educación y Ciencias Humanas, Nueva Etapa, 16(28), 162-163. Obtenido de http://www.postgrado.unesr.edu.ve/EYCH/template_2/PDF/10_Resena_Bibliografi ca.pdf

Murillo, J., \& et al. (2011). Evaluación Educativa para la Justicia Social. Revista Iberoamericana de Evaluación Educativa, 4(1), 7-23.

Not, L. (1992). La Enseñanza Dialogante. Barcelona: Editorial Herder.

Pérez, R. (2005). Calidad de la Educación: Hacia su Necesaria Integración. Educación XX1, 21(8), 11-33. Obtenido de http://revistas.uned.es/index.php/educacionXX1/article/view/341

Puig, J. (2007). Construcción Ideológica de la Personalidad Moral. Revista Iberoamericana de Educación, 8(1). Obtenido de http://rieoei.org/oeivirt/rie08a04.htm

Rueda, M., \& Díaz, F. (2011). La Evaluación de la Docencia en la Universidad. Perspectivas desde la investigación y la intervención profesional. México: Plaza y Valdés.

Rueda, M., Díaz, F., \& Díaz, M. (2001). Evaluar para Comprender y Mejorar la Docencia en la Educación Superior. México: UAM. CESU UNAM. UABJO.

Santos, M. (1993). La Evaluación: un proceso de diálogo, comprensión y mejora. Investigación en la Escuela(20), 23-35.

Scriven, M. (1967). La Metodología de la Evaluación. Perspectivas en evaluación y currículo. Chicago: Series de Monografías en Currículo y Evaluación AERA. 
Universidad de Antioquia. (s.f.). Ética como Amor Propio. Obtenido de aprendeenlinea.udea.edu.co:

http://aprendeenlinea.udea.edu.co $/ \mathrm{lms} / \mathrm{moodle} / \mathrm{mod} / \mathrm{resource} / \mathrm{view} \cdot \mathrm{php} ? \mathrm{id}=26908$

Velasco, J., \& Alonso, L. (2008). Sobre la Teoría de la Educación Dialógica. Revista Educere, 12(42), 461-468. Obtenido de http://www.redalyc.org/pdf/356/35614569006.pdf 